\title{
Cerebrospinal Fluid Leakage, CTCAE
}

National Cancer Institute

\section{Source}

National Cancer Institute. Cerebrospinal Fluid Leakage, CT CAE. NCI Thesaurus. Code C143361.

A disorder characterized by loss of cerebrospinal fluid into the surrounding tissues. 